\title{
Effective atomic orbitals for fuzzy atoms
}

\author{
I. Mayer ${ }^{1}$ and P. Salvador ${ }^{2, a)}$ \\ ${ }^{1}$ Chemical Research Center, Hungarian Academy of Sciences, P.O. Box 17, H-1525 Budapest, Hungary \\ ${ }^{2}$ Department of Chemistry and Institute of Computational Chemistry, University of Girona, \\ 17071 Girona, Spain
}

(Received 5 March 2009; accepted 22 May 2009; published online 18 June 2009)

\begin{abstract}
The method of extracting effective atomic orbitals and effective minimal basis sets from molecular wave function characterizing the state of an atom in a molecule is developed in the framework of the "fuzzy" atoms. In all cases studied, there were as many effective orbitals that have considerable occupation numbers as orbitals in the classical minimal basis. That is considered to be of high conceptual importance. (c) 2009 American Institute of Physics. [DOI: 10.1063/1.3153482]
\end{abstract}

\section{INTRODUCTION}

Our qualitative understanding of molecular wave functions relies mainly on the concept of minimal basis sets: we speak about the geometrical characteristics of $s p^{n}$ hybridized carbons, discuss the role of $d$ orbitals for second row elements, etc. The precise quantum chemical calculations apply larger and larger basis sets, not even always of atomic character. The results of such calculations are some energetic or geometric parameters, which are often difficult to interpret in simple chemical terms. For that reason it is of interest to elaborate techniques permitting to extract effective atomic (or group) orbitals by the a posteriori analysis of the actual molecular wave functions, which can be put into correspondence with the classical notions of electronic configuration. That had been achieved in the framework of the "Hilbertspace analysis" (i.e., analysis in which the atom is identified with the nucleus and the subspace of the basis orbitals centered on it) by requiring stationary properties of Mulliken's net atomic populations corresponding to some localized orbitals. $^{1,2}$ It has also been found ${ }^{2}$ that the orbitals obtained can be identified with (or, at least, are very closely related to) McWeeny's classical atomic natural hybrids. ${ }^{3}$ The numerical calculations showed that there is always as many effective atomic orbitals (AOs) with occupation numbers considerably differing from zero as orbitals contained in the classical minimal basis of the given atom. (Exceptions are the hypervalent atoms-such as six valent sulfur-for which some additional effective AOs with small but non-negligible occupation numbers reflecting backdonations to the $d$ orbitals are also obtained. ${ }^{1}$ ) We think that one cannot overestimate the conceptual importance of this conclusion.

The alternative to the Hilbert-space analysis is the analysis performed in the three-dimensional (3D) physical space, in which the atom is identified with the nucleus and a domain of the physical space around the nucleus. That domain can be defined either with strict boundaries like in Bader's "atoms in molecules" (AIM) theory ${ }^{4}$ or may lack them ("fuzzy" atoms). There are cases when the $3 \mathrm{D}$ analysis is the only

\footnotetext{
a) Author to whom correspondence should be addressed. Electronic mail: pedro.salvador@udg.edu.
}

possibility-e.g., plane wave bases are not atom centered at all, but the diffuse orbitals entering "augmented" basis sets are also lacking any pronounced atomic character. For that reason, we have developed here the 3D analog of the calculating effective atomic basis sets and realized it to different definitions of the fuzzy atoms. As a matter of fact, the equations necessary for such calculations have been already derived in Ref. 5 for Bader's atom framework and in Ref. 2 for the case of the fuzzy atoms. However, the first attempts to realize the scheme for the fuzzy atoms gave discouraging results. ${ }^{6}$ That was the case because the "cutoff" function ${ }^{2}$ was identified with the atomic weight function $w_{A}(\vec{r})$ of the fuzzy atom theory and only most recently have we realized on the basis of the more careful study of the relationships between the effective AOs and McWeeny's natural hybrids ${ }^{3}$ that one should use the square of the latter.

It might be worth to mention that for the particular case of single-determinant wave functions and of the use of Bader's AIM theory, the localized orbitals obtained here are identical to those originating from the so-called domain averaged Fermi hole (DAFH) analysis. ${ }^{7}$ This is a consequence of the facts that for disjoint domains one has $w_{A}^{2}(\vec{r})=w_{A}(\vec{r})$ and that for single determinants the exchange density can be expressed through the first order density matrix. In the DAFH analysis the orbitals of each fragment are further localized by "isopycnic transformation" and the resulting orbitals are investigated by visual inspection. In our approach we emphasize the relevance of the truncated localized orbitals (natural hybrids) for the characterization of the electronic state of the given atom within the molecule.

\section{FORMALISM}

As early as in 1960, McWeeny introduced the concept of "natural hybrid orbitals"3 which in the case of an orthonormalized basis diagonalize the atomic block of the density matrix D. In the general nonorthogonal case, they can be obtained by requesting stationary properties for Mulliken's net atomic populations corresponding to the individual effective AOs (hybrids). ${ }^{1,2}$ In any case the natural hybrids permit to express Mulliken's net atomic population, 


$$
\varrho_{A A}(\vec{r})=\sum_{\mu, \nu \in A} D_{\mu \nu} \chi_{\nu}^{*}(\vec{r}) \chi_{\mu}(\vec{r})
$$

in terms of natural hybrids $\varphi_{i}^{A}$ in a diagonal form:

$$
\varrho_{A A}(\vec{r})=\sum_{i} \nu_{i}^{A} \varphi_{i}^{A *}(\vec{r}) \varphi_{i}^{A}(\vec{r})
$$

Here $\varphi_{i}^{A}$ are the normalized atomic hybrids and $\nu_{i}^{A}$ are their occupation numbers summing up to Mulliken's net population of atom $A$.

In order to generalize that approach to the case of a 3D analysis, we shall start by introducing a non-negative weight function $w_{A}(\vec{r})$ for each atom and each point of the 3D space, which satisfies the requirement

$$
\sum_{A} w_{A}(\vec{r})=1
$$

everywhere. It is assumed that $w_{A}(\vec{r})$ is large "inside" of atom $A$ and small "outside." [In the special case of Bader's AIM theory, ${ }^{4} w_{A}(\vec{r})=1$ for points inside the "domain" of atom $A$ and $w_{A}(\vec{r})=0$ outside of it.]

Multiplying the electron density $\varrho(\vec{r})$ with the left-hand side of Eq. (3) once or twice, one can decompose the electron density in the sum of atomic or atomic and diatomic components,

$$
\varrho(\vec{r})=\sum_{A} \rho_{A}(\vec{r})=\sum_{A, B} \varrho_{A B}(\vec{r}) .
$$

The term $\varrho_{A A}(\vec{r})$ (case $A=B$ in the right-hand side) represents the 3D generalization of Mulliken's net atomic density. It integrates to the net atomic population defined in the framework of the fuzzy atom analysis. ${ }^{8}$ It simply equals to

$$
\varrho_{A A}(\vec{r})=w_{A}^{2}(\vec{r}) \varrho(\vec{r}),
$$

or, expanding the density $\varrho(\vec{r})$ in terms of the molecular orbitals (MOs) $\varphi_{i}$ :

$$
\varrho_{A A}(\vec{r})=2 \sum_{i}^{\text {occ. }} w_{A}^{2}(\vec{r}) \varphi_{i}^{*}(\vec{r}) \varphi_{i}(\vec{r}) .
$$

(For the sake of simplicity the case of a single-determinant wave function with doubly filled orbitals is considered.) If one introduces the normalized "intra-atomic" part $\varphi_{i}^{A}$ of the orbital $\varphi_{i}$ as

$$
\varphi_{i}^{A}(\vec{r})=\mathcal{N}_{i}^{A} w_{A}(\vec{r}) \varphi_{i}(\vec{r}),
$$

where $\mathcal{N}_{i}^{A}$ is a normalization constant, then the atomic density $\varrho_{A A}(\vec{r})$ has a diagonal form of Eq. (2) for any orthonormalized set of (canonic or localized) MOs $\varphi_{i}$. However, the orbitals $\varphi_{i}^{A}(\vec{r})$ are, in general, not orthogonal to each other; moreover, they are even not necessarily linearly independent.

It appears that there is a unique set of localized orbitals, different for each atom, for which the intra-atomic parts (effective AOs, hybrids) $\varphi_{i}^{A}$ of the orbitals are orthogonal to each other-similarly to their parent global MOs $\varphi_{i}$-and can be used in the expansion of type (2). To obtain them we form the Hermitian matrix $\mathbf{Q}$ with the elements

$$
Q_{i j}=\int w_{A}^{2}(\vec{r}) \psi_{i}^{*}(\vec{r}) \psi_{j}(\vec{r}) d v,
$$

where the orbitals $\left\{\psi_{i}\right\}$ represent the set of initial (e.g., canonic) MOs. It is easy to see that $Q_{i j}$ is nothing but the overlap integral of the (un-normalized) intra-atomic components of orbitals $\psi_{i}$ and $\psi_{j}$. We diagonalize matrix $\mathbf{Q}$ with the unitary matrix $\mathbf{U}$ :

$$
\mathbf{U}^{\dagger} \mathbf{Q U}=\mathbf{\Lambda}
$$

where $\Lambda=\operatorname{diag}\left\{\lambda_{1}, \lambda_{2}, \ldots\right\}$ is the diagonal matrix of the eigenvalues, and form the set of localized orbitals $\left\{\varphi_{i}\right\}$ as

$$
\varphi_{i}(\vec{r})=\sum_{j} U_{j i} \psi_{j}(\vec{r}) .
$$

Thus the requested normalized effective AOs (hybrids) $\varphi_{i}^{A}$ can be written as

$$
\varphi_{i}^{A}(\vec{r})=\frac{1}{\sqrt{\lambda_{i}}} w_{A}(\vec{r}) \varphi_{i}(\vec{r}) .
$$

All $\lambda_{i} \geq 0$ owing to the metric character of matrix $\mathbf{Q}$ and all $\lambda_{i} \leq 1$, as it originates from the norm of a function which is obtained by multiplying a normalized orbital with a weight function $w_{A}(\vec{r}) \leq 1$. The latter fact represents an obvious advantage with respect to the Hilbert-space analysis where the properties of the Mulliken populations do not guarantee the fulfillment of such a limitation. Orbitals with very small $\lambda_{i}$ values should be omitted, similarly to the practice of Löwdin's canonic orthogonalization. It follows from the eigenvalue equation (9) that these functions are orthonormalized. Owing to their definitions, they can be used to present the atomic density $\varrho_{A A}$ defined in Eq. (6) as

$$
\varrho_{A A}(\vec{r})=2 \sum_{i}^{\text {occ. }} \lambda_{i} \varphi_{i}^{A *}(\vec{r}) \varphi_{i}^{A}(\vec{r}),
$$

i.e., we get the form of Eq. (2) with the natural occupation numbers $\nu_{i}^{A}=2 \lambda_{i}$. They sum to the fuzzy atom net atomic population $^{8}$ of the atom in question.

\section{SOME FUZZY ATOM DEFINITIONS}

The atomic weights $w_{A}(\vec{r})$ above can be derived from several atomic definitions within the fuzzy atom framework. In the past we have made use of the simplest Becke atoms ${ }^{9}$ in order to show how bond orders, overlap populations, or energy components could be obtained for fuzzy atoms. The shape of such Becke atoms (the value of the atomic weights) is determined by a set of atomic radii and the so-called stiffness parameter. The ratio between the atomic radii of two atoms determines the relative size of the atomic basins and the stiffness parameter controls the shape of the cutoff profile, i.e., the decay of the atomic weight from the value of 1 at the nuclear position to zero at the position of the neighboring atoms. In his scheme ${ }^{9}$ (originally introduced for performing multicenter numerical integrations) a simple analytical polynomial is used to describe the cutoff profile and the stiffness parameter is simply related to the order of that polynomial. 
Even though Becke's atoms are only mathematical constructs, the values of physical quantities such as populations, bond orders, or energy components one can obtain by their use are quite reasonable provided a balanced set of atomic radii is used, for instance, those of Slater ${ }^{10}$ or Suresh and Koga. ${ }^{11}$ The main drawback associated with the use of a fixed set of covalent atomic radii is that the same atoms are treated on equal footing in different chemical environments, i.e., partial ionic character of atoms cannot be properly accounted for. One way to circumvent this problem is to determine the ratio of the atomic radii for each pair of chemically bonded atoms according to the position of the minima of the total density along the internuclear axis. ${ }^{8}$ Such a scheme, referred as Becke- $\rho$ in Ref. 12 and also used by Francisco et $a l .,{ }^{13}$ gives values for electron populations and bond orders very close to those obtained by applying the disjoint AIM atomic definition of Bader.

An alternative is the fuzzy atoms based on the use of promolecular densities such as classical Hirshfeld (or "stockholder") atoms. ${ }^{14}$ In this approach the atomic weight at a given point of the space is determined by the ratio

$$
w_{A}(\vec{r})=\frac{\rho_{A}^{0}(\vec{r})}{\sum_{B} \rho_{B}^{0}(\vec{r})},
$$

where $\rho_{A}^{0}(\vec{r})$ represents the promolecular density of atom $A$. Such promolecular densities are typically obtained from a free atom calculation and are usually spherically averaged functions centered at the atomic nuclei.

Hirshfeld atoms are probably more appealing than Becke's mathematical construct but they require the use of proper reference state atomic densities. Classical Hirshfeld atoms suffer from the same problems concerning a proper description of partial ionization as Becke's. Also, the resulting shape of the atoms in the molecule is strongly dependent on the choice of the promolecular state of the atom. The typical example of LiF, for which one can choose either neutral or ionic promolecular free atom densities, illustrates this problem. ${ }^{15}$

Recently an improvement of the classical Hirshfeld approach in a manner by which the partial ionization is taken into account in an ingenious manner has been proposed. The so-called iterative Hirshfeld (Hirshfeld-I) process $^{15}$ is derived from the minimization of the missing information subjected to the constrain that the promolecular atomic densities integrate to the same (usually fractional) number of electrons as do the atoms in the molecule. This is accomplished in an iterative fashion by defining the promolecular densities as a proper linear combination of neutral and ionic (again spherically averaged) free atom densities. (More precisely, the promolecular densities of free atoms are calculated by using those numbers of electrons which represent the closest upper and lower integers to the actual value of the atomic population.) It has been shown that the Hirshfeld-I process is convergent and also its solution is unique ${ }^{16}$ (for a given selection of the electronic state-including multiplicity-for the free neutral and ionized atoms). In this sense it represents a practical solution of the problem associated with the choice of the state of the reference free atoms. The Hirshfeld-I method tends to produce larger partial atomic charges (in absolute value) and smaller covalent bond orders compared to the classical Hirshfeld atoms. It has been pointed out recently that Hirshfeld-I charges reproduce quite well the electrostatic potential. ${ }^{17}$

We have made use of all the fuzzy atom definitions mentioned above in order to obtain effective AOs. We have observed that the different fuzzy atom definitions induce only minor qualitative differences in the shape of the hybrids, and no fundamental discrepancies on the number or chemically meaningful hybrids and their occupations have been observed.

\section{SAMPLE CALCULATIONS}

We have implemented the formalism described above for several weight functions $w_{A}(\vec{r})$ within the framework of the fuzzy atom formalism, namely, the simplest Becke, ${ }^{9}$ Becke with adjustment of atomic radii according to the topology of the density (Becke- $\rho$ ) ${ }^{12}$ the classical Hirshfeld, ${ }^{14}$ and the recent Hirshfeld- $\mathrm{I}^{15}$ approach. As an example we have obtained the atomic hybrids for the molecule of alaninewhich presents several types of $\mathrm{C}, \mathrm{O}$, and $\mathrm{H}$ atoms and one of nitrogen-for the different fuzzy atom schemes at the Hartree-Fock (HF) level.

The occupation numbers of the atomic hybrids for the different atoms of $\alpha$-alanine obtained at the RHF/cc-pVTZ level are shown in Fig. 1. The first observation is that only five hybrids are appreciably populated for all heavy atoms and just one in the case of hydrogens, once again recovering the picture of minimal basis from a quite large basis set containing up to $30 \mathrm{AOs}$ on each heavy atom. It can be also seen that the populations of the AOs do not exceed 2, as expected. This is not the case for Mulliken-type analysis where lone pairs often exhibit larger net populations, arising from small negative overlap populations with the neighboring atoms. In this sense, the present fuzzy atom approach seems more satisfactory from a conceptual point of view.

The chemical pictures obtained with the four fuzzy atom definitions are very similar. The drop on the occupation numbers is somewhat less pronounced in the case of Becke and classical Hirshfeld as compared to Becke- $\rho$ and Hirshfeld-I, for which the small $\nu_{i}^{A}$ values do not exceed 0.05. This can be explained by the fact that in the first two cases the ionic character of the atoms is not taken into account properly and the atomic weights are not adjusted to the actual situation in the molecule; therefore the atoms penetrate more into each other which leads to larger tails on the occupation number distribution of the hybrids.

Of course, some differences exist for the values of the occupation numbers of the orbitals, which are quite noticeable in the case of $\mathrm{H}$ atoms. For both Hirshfeld approaches the net occupations of the $\mathrm{H}$ atoms are below 0.6 and as low as 0.18 for the acidic $\mathrm{H}$ atom in the Hirshfeld-I case. This small net population is connected to the fact that in stockholder schemes the atoms are not "well cut" in the sense that the weight function of atom $A$ does not vanish at the nuclear position of atom $B$, as imposed by Becke atoms. This is particularly important when either $A$ or $B$ are hydrogen at- 

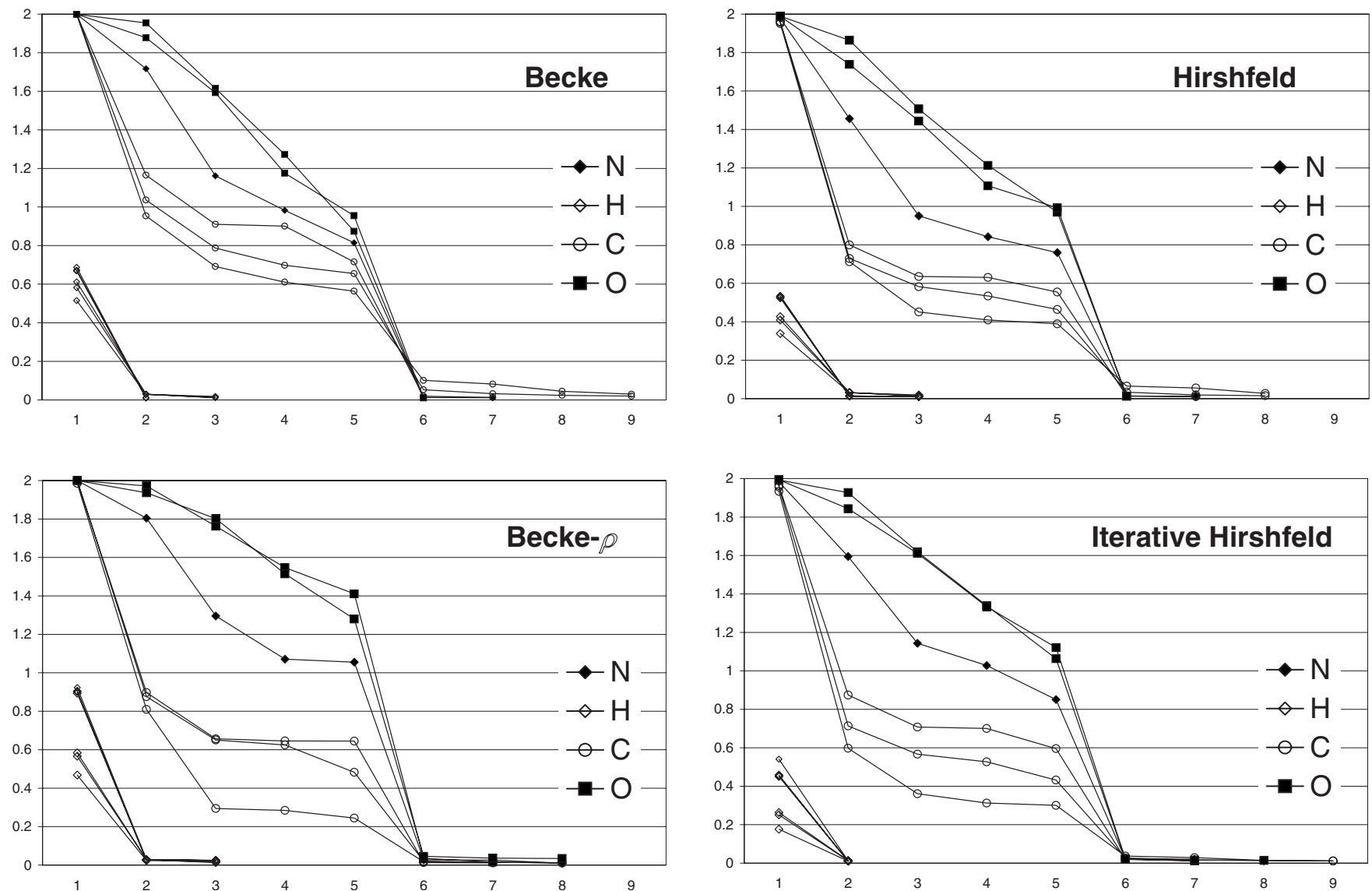

FIG. 1. Occupation numbers (in descending order) of the effective AOs of different atoms in $\alpha$-alanine molecule calculated by using cc-pVTZ basis set and four different fuzzy atom definitions.

oms. For instance, in the case of methane the weight of $\mathrm{C}$ atom amounts to typically $10 \%$ at the proton position. ${ }^{18}$

The values of the occupation numbers of the hybrids also discriminate between the same atoms in different chemical environments. The hydrogen atoms with less net occupations are the acidic proton of the carboxylic group, followed by the two amine protons. This is observed in all fuzzy atom definitions. The populations of the carboxylic $\mathrm{C}$ atom are always systematically smaller than those of the other two aliphatic carbons. In the particular case of Becke- $\rho$ and Hirshfeld-I the population of the $s p^{2}$ hybrids barely reaches 0.30 as a direct consequence of the very large partial atomic charge of the atom (up to +1.7 and +1.1 , respectively). Large charges such as those provided by the Becke- $\rho$ method may be expected in Bader's framework, too.

We have carried out the calculations also for smaller basis sets such as the 6-31G and also for basis sets with somewhat less atomic character (containing diffuse functions) such as the $6-31++\mathrm{G}^{* *}$ one. As might be anticipated for a 3D-space based analysis, the results obtained do not differ significantly. For instance, it is remarkable that the largest discrepancy in the occupation numbers between orbitals obtained from the quite different cc-pVTZ and 6-31G basis sets is only 0.10 , which refers to an $s p$ hybrid of the $\mathrm{N}$ atom.

For illustration purposes we provide in Fig. 2 surface plots of the five populated atomic hybrids of the hydroxylic oxygen obtained using the Hirshfeld-I fuzzy atom scheme.
One can clearly identify a $1 s$-type core orbital, a classical lone pair, a $p$ orbital of the local $\pi$ system, and two hybrids of $\sigma$ character oriented toward the hydrogen and the carboxylic carbon atom, respectively. The occupation numbers of these hybrids are $1.99,1.84,1.61,1.33$, and 1.12 , respectively, showing that the electrons on the core and lone pair orbitals are practically not shared with the neighboring at-
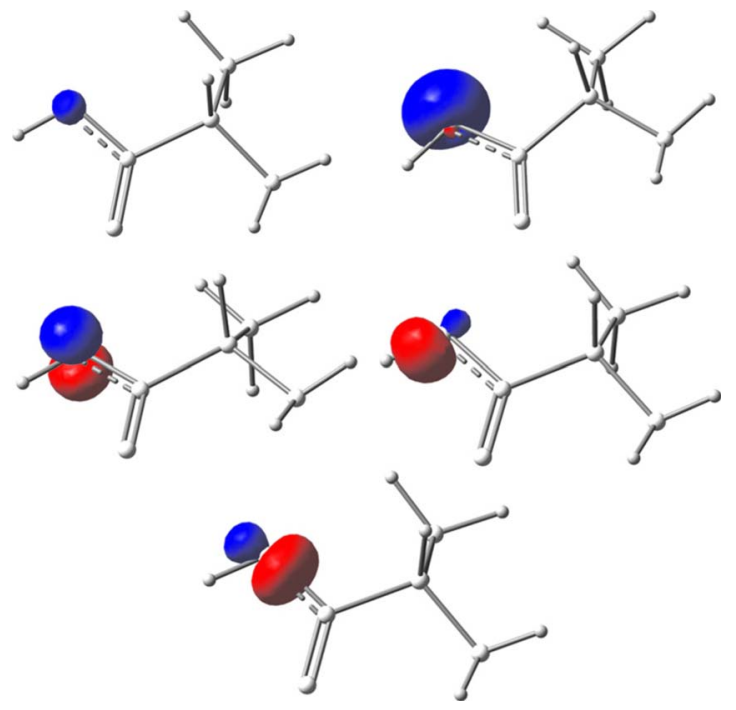

FIG. 2. (Color online) Effective AOs of the hydroxylic oxygen atom in $\alpha$-alanine molecule calculated by using cc-pVTZ basis set and the Hirshfeld-I fuzzy atom definitions. 


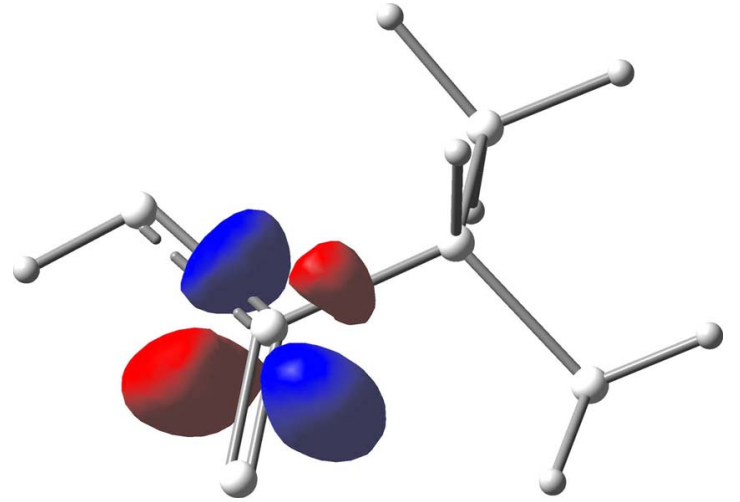

FIG. 3. (Color online) The sixth (weakly populated) effective AO of the carboxylic carbon atom in $\alpha$-alanine molecule calculated by using cc-pVTZ basis set and the Hirshfeld-I fuzzy atom definitions.

oms. The shapes of the orbitals obtained from the other fuzzy atom definitions are very similar and are not reported. The only appreciable differences are related to the extent of penetration of the orbitals into the vicinity of the neighboring atoms, which is essentially determined by the shape of the respective atomic cutoff function. In particular, the Becke weight functions diminish much faster than do the Hirshfeld ones.

Even though the atomic hybrids with low occupations have generally no chemical significance, sometimes they do bear some chemical relevance. This is the case, for instance, of the sixth orbital of the carboxylic $\mathrm{C}$ atom, for which the occupation number ranges between 0.04 and 0.10 for Hirshfeld-I and Becke, respectively. In Fig. 3 we can see that this orbital is clearly of $d$ type and accounts for a minor backdonation from the oxygen atoms to the very positive carbon. That seems similar to that observed for the hypervalent sulfur atoms (vide infra).

We have also considered the series of sulfur compounds $\mathrm{SF}_{2}, \mathrm{SF}_{4}$, and $\mathrm{SF}_{6}$. The occupations (above 0.05) of the atomic hybrids obtained for both the $\mathrm{S}$ and $\mathrm{F}$ atoms using the Hirshfeld-I method are listed in Table I. In $\mathrm{SF}_{2}$ there are seven orbitals for the $\mathrm{S}$ atom with large occupation numbers (above 1.7); for $\mathrm{SF}_{4}$ there are six and for $\mathrm{SF}_{6}$ only five. This is indicative of the presence of two lone pairs for $\mathrm{SF}_{2}$, one lone pair for $\mathrm{SF}_{4}$, and none for $\mathrm{SF}_{6}$ (the first five natural orbitals correspond to the core orbitals). The remaining valence orbitals for $\mathrm{S}$ present occupation numbers in the range $0.3-0.5$ in all cases. Such rather small values are due to the strong electron withdrawing force of the $\mathrm{F}$ atoms. There is a clear gap in the occupation numbers of the $\mathrm{S}$ atom. In $\mathrm{SF}_{2}$ this occurs after the last valence orbital, whereas for $\mathrm{SF}_{4}$ and $\mathrm{SF}_{6}$ one and two (degenerate) more natural AOs are significantly populated ( 0.173 and 0.130 , respectively). These orbitals have a clear $d$ character and correspond to some backdonation from the $\mathrm{F}$ atoms to the sulfur, which is important to understand the electronic structure of such hypervalent compounds. ${ }^{1}$ The occupation numbers of the $\mathrm{F}$ atoms are quite similar in these systems: in all cases only five of them have significant values.

The picture obtained with the other fuzzy atom schemes is quite similar. Hirshfeld and Hirshfeld-I give very close results; Hirshfeld-I gives somewhat larger polarization, as expected. In the case of the simple Becke scheme one meets the difficulty of a too small fluorine radius already discussed in Ref. 8, leading to unphysical negative sulfur charges. With the use of the Becke- $\rho$ scheme the atomic charge of the F atoms is always close to -1 , which makes the $\mathrm{S}$ atom extremely positive, with charges $+2.0,+3.4$, and +4.4 in the series. This may be considered somewhat exaggerated, as these values exceed even the AIM ones. ${ }^{19,20}$

\section{CONCLUSIONS}

The method of extracting effective AOs and effective minimal basis sets from molecular wave function is developed in the framework of the fuzzy atoms. In numerical test calculations, several weight functions defining the fuzzy atoms have been considered. The chemical pictures obtained with the different definitions are very similar: in all cases studied, there are as many effective orbitals that have con-

TABLE I. Occupation numbers (above 0.05) of the effective AOs of sulfur and fluorine in the molecules $\mathrm{SF}_{x}$, $x=2,4,6$, calculated at the HF/cc-pVTZ level and Hirshfeld-I atom definition.

\begin{tabular}{|c|c|c|c|c|c|c|}
\hline \multicolumn{2}{|c|}{$\mathrm{SF}_{2}$} & \multicolumn{3}{|c|}{$\mathrm{SF}_{4}$} & \multicolumn{2}{|c|}{$\mathrm{SF}_{6}$} \\
\hline S & $\mathrm{F}$ & S & $\mathrm{F}_{\mathrm{eq}}$ & $\mathrm{F}_{\mathrm{ax}}$ & $\mathrm{S}$ & $\mathrm{F}$ \\
\hline 2.000 & 1.997 & 2.000 & 1.999 & 1.998 & 1.999 & 1.998 \\
\hline 1.996 & 1.940 & 1.988 & 1.965 & 1.951 & 1.963 & 1.957 \\
\hline 1.993 & 1.796 & 1.977 & 1.834 & 1.786 & 1.963 & 1.802 \\
\hline 1.987 & 1.745 & 1.976 & 1.811 & 1.767 & 1.963 & 1.802 \\
\hline 1.983 & 1.267 & 1.969 & 1.400 & 1.279 & 1.962 & 1.302 \\
\hline 1.873 & & 1.647 & & & 0.557 & \\
\hline 1.767 & & 0.438 & & & 0.313 & \\
\hline 0.444 & & 0.344 & & & 0.313 & \\
\hline 0.358 & & 0.325 & & & 0.313 & \\
\hline 0.080 & & 0.138 & & & 0.130 & \\
\hline 0.057 & & 0.065 & & & 0.130 & \\
\hline \multirow[t]{3}{*}{0.055} & & 0.058 & & & 0.056 & \\
\hline & & 0.053 & & & 0.056 & \\
\hline & & & & & 0.056 & \\
\hline
\end{tabular}


siderable occupation numbers as orbitals in the classical minimal basis, which is considered to be of high conceptual importance.

\section{ACKNOWLEDGMENTS}

This work has been supported by Project No. PCI2006A7-0631 in the framework of the International Cooperation program of the Spanish Ministerio de Ciencia e Innovacion. I.M. acknowledges the partial support by the Hungarian Scientific Research Fund (Grant No. OTKA 71816). P.S. also acknowledges partial support from the Spanish Ministerio de Educacion y Ciencia (Project No. MAT2008-04834/MAT).

${ }^{1}$ I. Mayer, Chem. Phys. Lett. 242, 499 (1995).

${ }^{2}$ I. Mayer, J. Phys. Chem. 100, 6249 (1996).

${ }^{3}$ R. McWeeny, Rev. Mod. Phys. 32, 335 (1960).

${ }^{4}$ R. F. W. Bader, Atoms in Molecules: A Quantum Theory (Oxford University Press, Oxford, 1990).
${ }^{5}$ I. Mayer, Can. J. Chem. 74, 939 (1996).

${ }^{6}$ I. Mayer (unpublished).

${ }^{7}$ R. Ponec, J. Math. Chem. 21, 323 (1997).

${ }^{8}$ I. Mayer and P. Salvador, Chem. Phys. Lett. 383, 368 (2004).

${ }^{9}$ A. D. Becke, J. Chem. Phys. 88, 2547 (1988).

${ }^{10}$ J. C. Slater, J. Chem. Phys. 41, 3199 (1964).

${ }^{11}$ C. H. Suresh and N. Koga, J. Phys. Chem. A 105, 5940 (2001).

${ }^{12}$ E. Matito, M. Solà, P. Salvador, and M. Duran, Faraday Discuss. 135, 325 (2007).

${ }^{13}$ E. Francisco, A. Martin-Pendás, and M. A. Blanco, J. Chem. Theory Comput. 2, 90 (2006).

${ }^{14}$ F. L. Hirshfeld, Theor. Chim. Acta 44, 129 (1977).

${ }^{15}$ P. Bultinck, C. Van Alsenoy, P. W. Ayers, and R. Carbó-Dorca, J. Chem. Phys. 126, 144111 (2007).

${ }^{16}$ P. Bultinck, P. W. Ayers, S. Fias, K. Tiels, and C. Van Alsenoy, Chem. Phys. Lett. 444, 205 (2007).

${ }^{17}$ S. Van Damme, P. Bultinck, and S. Fias, J. Chem. Theory Comput. 5, 334 (2009).

${ }^{18}$ I. Mayer, Phys. Chem. Chem. Phys. 8, 4630 (2006)

${ }^{19}$ K. C. Lobring, C. E. Check, T. M. Gilbert, and L. S. Sunderlin, Int. J. Mass. Spectrom. 227, 361 (2003).

${ }^{20}$ J. Cioslowski and S. T. Mixon, Inorg. Chem. 32, 3209 (1993). 